\title{
Die Refluxkrankheit kommt und geht
}

\section{Die gastroösophageale Refluxkrank- heit (GERD) wird absolut immer häu- figer, so das Ergebnis einer großen Kohortenstudie aus Skandinavien. Die gute Nachricht: Spontane Hei- lungen sind nicht selten.}

- Die gastroösophageale Refluxkrankheit ist außerordentlich häufig. Dennoch gibt es kaum valide Daten zum Spontanverlauf. Es wird angenommen, dass die Krankheit häufiger geworden ist, und dass sie praktisch immer chronisch verläuft.

In einer populationsbasierten Kohortenstudie (HUNT Studie) in Norwegen wurden 1995-1997 insgesamt 58869 (Response Rate 64\%) und 2006-2009 erneut 44997 Erwachsene (Response Rate 49\%) hinsichtlich ihrer Refluxsymptome in den vorangegangenen zwölf Monaten befragt. Insgesamt konnten 29610 Personen prospektiv über elf Jahre nachbeobachtet werden.
Von 1995-1997 bis 2006-2009 stieg die Prävalenz von Refluxsymptomen insgesamt von $31,4 \%$ auf $40,9 \%$, die von schweren Refluxsymptomen von 5,4\% auf $6,7 \%$ und die von zumindest einmal wöchentlichen Symptomen von 11,6\% auf $17,1 \%$.

Die jährliche Inzidenz von Refluxsymptomen lag bei $3,07 \%$, und die von schweren Refluxsymptomen bei $0,23 \%$. Nur bei Frauen nahm die Inzidenz der Refluxsymptome mit dem Alter zu.

Bei durchschnittlich 2,32\% aller Personen mit Refluxsymptomen und bei $1,22 \%$ derjenigen mit schweren Symptomen sistierten die Beschwerden anhaltend spontan. Die Konversion symptomatisch $\mathrm{zu}$ asymptomatisch wurde mit zunehmendem Alter seltener.

\footnotetext{
- E. Ness-Jensen et al.

Changes in prevalence, incidence and spontaneous loss of gastro-oesophageal reflux symptoms. Gut 2012;61:1390-1397
}

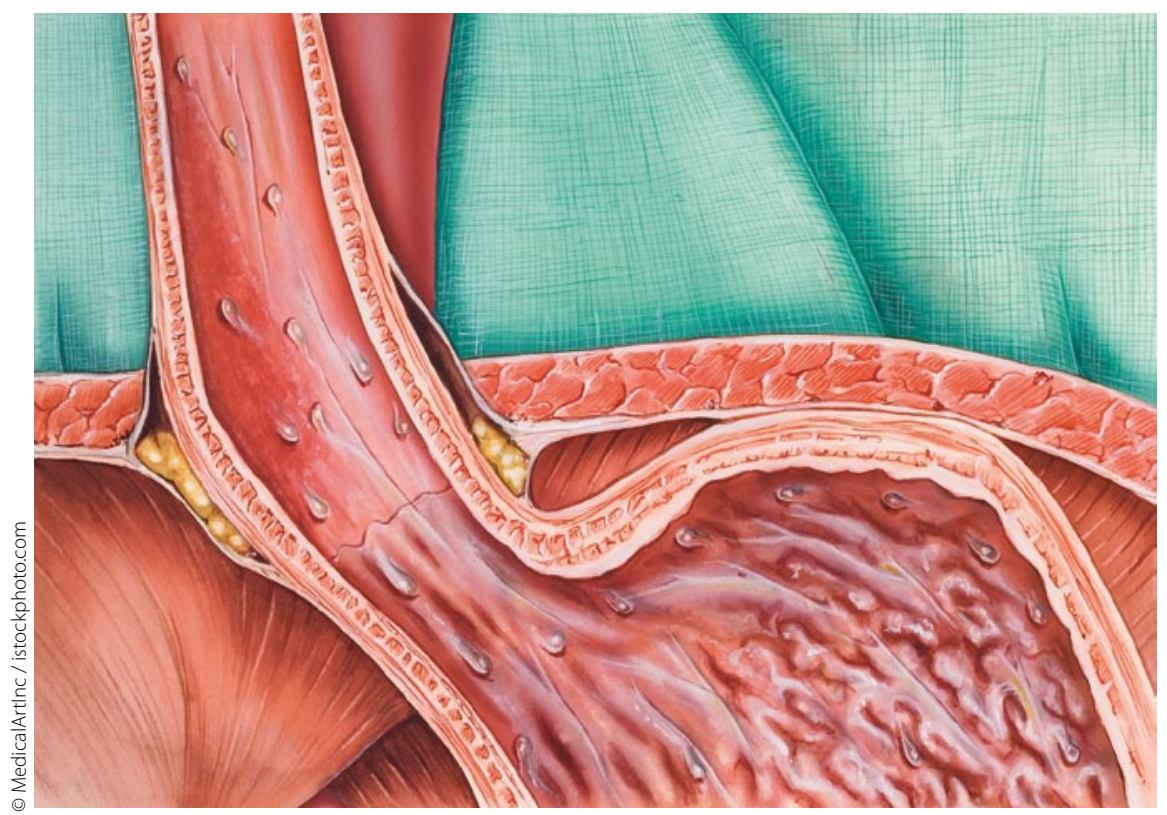

Eine unbehandelte Refluxerkrankung kann auch spontan dauerhaft abheilen.

\section{Kommentar}

Die GERD ist aktuell wohl die häufigste gastroenterologische Erkrankung. Sie verursacht erhebliche Kosten, insbesondere für die medikamentöse Behandlung. Gute epidemiologische Studien zur GERD gibt es kaum. Vor diesem Hintergrund ist diese große und populationsbasierte Studie von enormem wissenschaftlichem Wert. Sie bestätigt das, was wir in der täglichen Praxis gefühlt haben und was Studien minderer Qualität aus verschiedenen Regionen der Erde signalisierten: Die Krankheit wird absolut häufiger. Über die Gründe hierfür kann nur spekuliert werden: Zunahme der Adipositas und Abnahme der H.-pylori-Durchseuchung mögen eine Rolle gespielt haben.

Sie zeigt aber auch - und das war in diesem Umfang so nicht bekannt - Spontanheilungen bei einem substanziellen Anteil der Patienten. Dies hat natürlich Einfluss auf das Patientenmanagement. Die Möglichkeit der anhaltenden Symptombefreiung auch ohne spezifische Medikation sollte in das Therapiegespräch einbezogen werden. Auch sollte man eine medikamentöse Therapie - zumindest bei Patienten mit GERD oder leichter Ösophagitis - a priori zeitlich befristen. Dies beugt einer PPI-Abhängigkeit vor, die vermutlich der wesentliche Grund für die hohe Anzahl an Langzeittherapien und dem insgesamt drastisch gestiegenen PPIVerbrauch ist.

Da Schweregrad und Häufigkeit von Refluxsymptomen so gut wie keine Korrelation zu endoskopisch detektierbaren Läsionen aufweisen, kann aus den Studiendaten nicht auf den Spontanverlauf ösophagealer Schäden geschlossen werden. Auch muss bei der Betrachtung der Epidemiologie berücksichtigt werden, dass in einer skandinavischen Populationsstudie (KALIXANDA) immerhin 6\% der erwachsenen Bevölkerung endoskopisch eine asymptomatische Refluxösophagitis aufweisen. Der Spontanverlauf von refluxbedingten Schäden in der Speiseröhre bleibt weiterhin unklar.

J. LABENZ 